\title{
NOUVELLE
}

\section{Stress oxydatif \\ et prédiction des accidents cardiovasculaires}

Raymond Ardaillou

malades qui sont morts, ont eu un infarctus ou ont dû subir une intervention pour revascularisation. Comme pour la glutathion peroxydase, l'activité de la myéloperoxydase a une

Inserm U.489, Hôpital Tenon, 4 , rue de la Chine, 75970 Paris Cedex 20, France. raymond.ardaillou@academie-medecine.fr
> Les formes actives de l'oxygène (anion superoxyde, peroxyde d'hydrogène, peroxynitrites) sont impliquées à toutes les phases du développement de la plaque d'athérome depuis le dépôt initial de lipides jusqu'à la rupture de plaque et à la thrombose. Le stress oxydatif est la conséquence du déséquilibre entre production et destruction de ces métabolites. Il était donc logique de rechercher si l'augmentation de l'activité des enzymes productrices et la diminution de celle des enzymes destructrices n'étaient pas prédictives d'accidents cardiovasculaires chez les sujets à risque. C'est ce qu'ont tenté de montrer S. Blankenberg et al. [1] pour la glutathion peroxydase, enzyme ubiquitaire inactivant le peroxyde d'hydrogène, et M.L. Brennan et al. [2] pour la myéloperoxydase, enzyme leucocytaire produisant le peroxyde d'hydrogène.

Dans la première étude, les auteurs ont suivi pendant 4,7 ans en moyenne une cohorte de 636 malades ayant consulté pour des symptômes d'insuffisance coronarienne, confirmée par coronarographie chez 510 d'entre eux. Soixantequatre moururent d'accident cardiovasculaire et 19 eurent un infarctus du myocarde non létal. À leur entrée dans l'étude, le dosage de l'activité de la glutathion peroxydase 1 dans les hématies a été effectué. II s'est avéré que la diminution de cette activité était fortement associée au risque d'accident cardiovasculaire puisque les malades dont l'activité enzymatique se trouvait dans le quartile le plus élevé présentaient un risque cardiovasculaire inférieur de plus de trois fois $(0,29$ en moyenne) à celui des patients dont l'activité enzymatique se situait dans le quartile le plus bas. Les auteurs ont constaté que l'activité de la glutathion peroxydase 1 avait une valeur prédictive indépendante des autres paramètres biologiques associés au risque cardiovasculaire (LDL cholestérol, homocystéine), des paramètres inflammatoires (protéine $C$-réactive), et des caractéristiques cliniques (âge, fraction d'éjection ventriculaire, douleurs d'effort ou au repos). L'activité de la glutathion peroxydase 1 était plus basse chez les fumeurs et plus élevée chez les femmes jeunes, mais conservait sa valeur prédictive après ajustement pour ces variables. Un argument supplémentaire en faveur de ce paramètre est la fiabilité et la reproductibilité de sa mesure.

Dans la deuxième étude, 604 malades hospitalisés dans un service d'urgence pour des douleurs thoraciques ont été comparés à 115 sujets témoins. L'évolution de la maladie chez les premiers a été appréciée avec un recul de 30 jours et 6 mois. L'activité de la myéloperoxydase dans le plasma était en moyenne plus élevée chez les malades (198 pM) que chez les témoins (120 pM). Le résultat le plus frappant fut le parallélisme entre l'accroissement du risque d'infarctus du myocarde et celui de l'activité enzymatique, passant de $13,9 \%$ dans le premier quartile $(<119,4 \mathrm{pM})$ à $38,4 \%$ dans le dernier quartile (>394 pM). Plus généralement, l'activité de la myéloperoxydase était plus élevée chez les valeur prédictive indépendante d'autres facteurs de risque (âge, sexe, hyperlipidémie, signes électrocardiogra-

phiques). Les auteurs ont étudié la relation entre sensibilité (d'autant meilleure que le test est positif chez les malades) et spécificité (d'autant meilleure que le test est négatif chez les sujets sains) à différentes valeurs de seuil, le test idéal étant celui qui allie bonne sensibilité et large gamme de spécificité. La myéloperoxydase répond à ce critère bien mieux que la troponine, l'isoforme du muscle cardiaque de la créatine kinase, ou la protéine C-réactive, même chez les malades sans nécrose myocardique initiale (ayant donc un taux de troponine normal).

Ces deux études suggèrent le rôle des formes actives de l'oxygène dans le mécanisme des accidents cardiovasculaires chez les coronariens. Cependant, la confirmation décisive d'une relation de cause à effet, c'est-à-dire la constatation d'une amélioration de l'évolution de la maladie après le changement expérimental de la valeur du marqueur (augmentation pour la glutathion peroxydase $l$ et diminution pour la myéloperoxydase) est toujours manquante. $\diamond$ Oxidative stress and prediction of coronary artery disease

\section{RÉFÉRENCES}

1. Blankenberg S, Rupprecht HJ, Bickel C, et al. Glutathione peroxidase $\mathrm{l}$ activity and cardiovascular events in patients with coronary artery disease. N Engl J Med 2003; 349: 1605-13.

2. Brennan ML, Penn MS, Van Lente F, et al. Prognostic value of myeloperoxidase in patients with chest pain. N Engl J Med 2003; 349: 1595-604. 